\title{
Observation of Magnetic reconnection at a 3D null point associated with a solar eruption
}

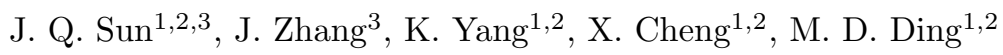 \\ ${ }^{1}$ School of Astronomy and Space Science, Nanjing University, Nanjing 210023, China \\ ${ }^{2}$ Key Laboratory for Modern Astronomy and Astrophysics (Nanjing University), Ministry of Education, \\ Nanjing 210023, China \\ ${ }^{3}$ Department of Physics, Astronomy and Computational Sciences, George Mason University, 4400 \\ University Drive, MSN 6A2, Fairfax, Virginia 22030, USA \\ dmd@nju.edu.cn; jzhang7@gmu.edu
}

\begin{abstract}
Magnetic null has long been recognized as a special structure serving as a preferential site for magnetic reconnection (MR). However, the direct observational study of MR at null-points is largely lacking. Here, we show the observations of MR around a magnetic null associated with an eruption that resulted in an M1.7 flare and a coronal mass ejection. The GOES Xray profile of the flare exhibited two peaks at $\sim 02: 23$ UT and $\sim 02: 40$ UT on 2012 November 8 , respectively. Based on the imaging observations, we find that the first and also primary Xray peak was originated from $\mathrm{MR}$ in the current sheet underneath the erupting magnetic flux rope (MFR). On the other hand, the second and also weaker X-ray peak was caused by MR around a null-point located above the pre-eruption MFR. The interaction of the null-point and the erupting MFR can be described as a two-step process. During the first step, the erupting and fast expanding MFR passed through the null-point, resulting in a significant displacement of the magnetic field surrounding the null. During the second step, the displaced magnetic field started to move back, resulting in a converging inflow and subsequently the MR around the null. The null-point reconnection is a different process from the current sheet reconnection in this flare; the latter is the cause of the main peak of the flare, while the former is the cause of the secondary peak of the flare and the conspicuous high-lying cusp structure.
\end{abstract}

Subject headings: Sun: flares Sun: coronal mass ejections (CMEs) Sun: magnetic topology

Online-only material: animations, color figures

\section{INTRODUCTION}

Magnetic reconnection (MR) has long been recognized as the principal physical mechanism responsible for solar flares. Where and how it takes place remains so essential in understanding the triggering and evolution of flares and associated coronal mass ejections (CMEs). The dual phenomena of flares and CMEs can be collectively called a solar eruption, because of close time coincidence between the impulsive phase of flares and strong acceleration phase of CMEs, both of which are likely driven by MR (Zhang et al. 2001). Over the past several decades, the flare-producing MR has been intensively studied and succinctly described 
with a standard 'CSHKP' flare model (Svestka \& Cliver 1992) (CSHKP refers to the studies of Carmichael 1964: Sturrock 1966 Hirayama 1974 Kopp \& Pneuman 1976). This model, consisting of a magnetic flux rope (MFR) and an underneath current sheet (CS) (Lin \& Forbes 2000), has been largely validated by many observational studies that showed consistent features, such as plasma inflows (Yokoyama et al. 2001; $\mathrm{Su}$ et al. 2013: Sun et al. 2015, Zhu et al. 2016), outflows or down flows (McKenzie \& Hudson 1999: Liu et al. 2013), loop-top hard X-ray sources(Masuda et al. 1994), coronal X-ray sources (Sui \& Holman 2003), the termination shock (Chen et al. 2015), and so on. The CSHKP model has been successful in explaining a large array of observational features associated with eruptive flares. Nevertheless, it is largely a two-dimensional (2D) model and has a simple bipolar magnetic configuration. It has an intrinsic shortage in describing certain realistic solar flare events, which may explicitly involve the three-dimensional (3D) structure in the modeling in order to understand certain observational features.

One of the important subjects in 3D MR theories concerns the peculiar structure of null-points. Previous theoretical studies suggest that 3D null-point may be a preferential site for the generation of MR (Priest) \& Titov 1996, Priest \& Pontin 2009). Based on the theoretical models, a series of numerical experiments are conducted through applying perturbations, like rotational and shearing motions, to the spine and fan structures extended from the null. As a result, strong current is found to develop around the null-point (Pontin \& Galsgaard 2007; Galsgaard \& Pontin 2011, Wyper et al. 2012). Pontin et al. (2013) demonstrated that the connectivity of the magnetic field line would change when it transfers across the CS forming around the null-point and treat that as a feature of spine-fan reconnection. In addition, the role of 3D reconnecting null-point in accelerating particles is also confirmed by several simulations (Browning et al. 2010, Stanier et al. 2012, Baumann et al. 2013a|b). However, in observations, the reconnection around null-point has only been reported in a handful of studies in the Sun (Filippov 1999, Fletcher et al. 2001, Sun et al. 2012, Wang \& Liu 2012, Sun et al. 2014a) and in the Earth's magnetosphere (Xiao et al. 2006). The detailed evolution of the magnetic field and plasma surrounding the null-point during the reconnection, i.e. the observational features of the null-point reconnection, has been seldom obtained. Such lack of observational studies restrict the improvement of theoretical models. Consequently, how the null-point reconnection is triggered and conversely how it affects the evolution of flares are still open questions that need more observations to answer (Török et al. 2011).

Here, we show the direct observations that reveal the processes of how MR is triggered and how it proceeds at a null-point. The observations are based on an array of instruments, including the Solar Dynamics Observatory (SDO; Pesnell et al. 2012), Reuven Ramaty High Energy Solar Spectroscopic Imager (RHESSI; Lin et al. 2002), Nobeyama Radioheliograph (NoRH; Nakajima et al. 1994), and Geostationary Operational Environmental Satellites (GOES). The overall observations are presented in $\S 2.1$. 22.2 focuses on the process of $\mathrm{MR}$, followed by the analysis of the $3 \mathrm{D}$ magnetic topology in $\S 2.3$. We conclude the results and discuss their significance in $\S 3$.

\section{Results}

\subsection{Observations of the flare}

On 2012 November 8, a GOES M1.7-class flare occurred near the northeastern solar limb. From the GOES 1-8 $\AA$ soft X-ray (SXR) flux profile, the flare started at $\sim 02: 10$ UT and peaked at $\sim 02: 23$ UT. The gradual declining of the flux was interrupted by a second enhancement at $\sim 02: 38$ UT and reached a second peak at $\sim 02: 40$ UT (Figure 2(c)). This double-peak property of the flare emission is more obvious in the 
profiles of Atmospheric Imaging Assembly (AIA; Lemen et al. 2012) $94 \AA$ and $131 \AA$ fluxes (Figure 2(c)). Prior to the SXR increase, a filament already began to rise up slowly, producing some sporadic brightenings along the filament structure (Figure 1(a)-(b)). As the slow-rising motion of the filament continued, a 6-12 $\mathrm{keV}$ X-ray source appeared slightly underneath it at 02:08 UT and was quickly evolved to contain higher energy emission of $12-25 \mathrm{keV}$ at 02:10 UT. About one minute later, probably due to plasma heating, the upper part of the filament disappeared in $304 \AA$ and $171 \AA$ images; instead, an 'M' shaped hot structure appeared there in $131 \AA$ passband (Figure 1(c)). This hot structure is likely to be the core part of the erupting MFR. Under the MFR, a post-flare loop arcade appears in $131 \AA$ passband (Figure 1(c)-(d)). At the same time, the 25-50 keV non-thermal HXR sources resulting from the accelerated electrons are found at two footpoints and above the top of the loop. These observational features are consistent with the classical flare models that MR takes place in a vertical CS located between the CME and the post-flare loops.

Following the eruption, the flare soon achieved the SXR peak at 02:23 UT and evolved into the gradual phase. However, the gradual decay of the SXR emission just lasted for a short time; 15 minutes later, at $\sim 02: 38$ UT, the SXR began to increase again and reached a second peak at $~ 02: 40$ UT. Correspondingly, there also existed a two-stage energy release in HXR, appearing as two independent bumps in Fermi HXR spectrogram (Figure 2(a)). The second bump appeared after 02:35 UT and was less energetic (mainly under $20 \mathrm{keV}$ ) than the first one in the impulsive phase (up to more than $100 \mathrm{keV}$ ). In AIA $193 \AA$ images, a new southeast-directed cusp appeared at a higher altitude after 02:40 UT (Figure 1(f)). We chose two slices, i.e. Slice 1 and Slice 2 as plotted in Figure 2(d), along and perpendicular to the cusp, respectively, to extract the time-distance evolution maps of the cusp. Along the cusp, we find two above-the-loop-top hot regions $(\geq 10$ MK) (Figure 2(b)) that appeared successively in time, coinciding temporally with the SXR double peaks. Hot region 1 appeared in the impulsive phase of the flare at a height of $\sim 10 \mathrm{Mm}$. Prior to its disappearance at $\sim 02: 36 \mathrm{UT}$, it continuously moved upward for $\sim 10 \mathrm{Mm}$. Hot region 2 emerged at a higher altitude of $\sim 22$ $\mathrm{Mm}$. However, the location of hot region 2 almost remained stationary during its $\sim 5$ minutes of apparent life. All the observations from EUV to HXR show the evidence of a second energy release, which originated from a different location and had different properties from the one in the first and main peak of the flare.

\subsection{The $3 \mathrm{D}$ null-point magnetic reconnection}

In the impulsive phase, the main features of the flare, including the outward eruption bubble, the underneath flare loop arcade, the coronal and footpoint HXR sources, and the rising above-the-loop-top hot region, can be explained by the CSHKP model. Due to the ascent of the presumed MFR, the reconnection site in the vertical CS is also expected to move upward, causing the rise of the flare loop arcade and the hot region 1 (Figure 2(b)).

However, the CSHKP model could not explain the second peak, which appeared in the gradual phase. In $\S 2.1$, we have already shown some evidence of MR responsible for the second peak, such as a higher southeast-directed cusp, an above-the-loop-top hot region as well as the emission enhancement from EUV to HXR. In the following, we focus on some dynamic properties of the second-peak reconnection, i.e. where and how magnetic fields reconnect. It is found that the evolution of the magnetic field participating in such a reconnection can be divided into two steps: (1) the separation distortion, and (2) the recovery inflow leading to the MR. The outward-moving and expanding MFR distorted the surrounding coronal fields, in particular, caused a large separation displacement of the overlying magnetic fields along the direction of slice 2 from 02:10 UT to 02:20 UT (Figure 3(d)). The departure of the MFR from the flare region left an area of low magnetic pressure behind it. Due to the required pressure balance with the surroundings, the corona loops 
pushed aside during the eruption, denoted as 'Coronal loops S' (CLS) and 'Coronal loops N' (CLN) in Figure $1(\mathrm{~g})$, began to recover and approach each other; the approaching or inflow velocity is in a range of $1-10 \mathrm{~km}$ $\mathrm{s}^{-1}$ at $\sim 03: 03 \mathrm{UT}$, calculated from the Fourier local correlation tracking (FLCT) method (Figure 3(a)). The inflow formed an X-shaped structure seen in $171 \AA$ images. According to the frozen-in property in a highly conductive plasma, these observed loops may well trace out the magnetic field lines in the corona, thereby indicating an X-shaped magnetic topology there. Once the CLS and CLN contacted with each other at the X-point (the center of the X-shaped structure, marked by an orange cross in Figure 3(b)-(c)), they suddenly disappeared (Figure 3(d)). Immediately afterward, a cusp structure (Figure 1(h)) was formed and lightened up in the $131 \AA$ passband under the X-point. With the AIA multi-wavelength observations, we calculate the differential emission measure (DEM) using the method from Hannah \& Kontar (2012). We define the total emission measure (EM) and DEM-weighted temperature $(\mathrm{T})$ as:

$$
E M=\int_{T_{\min }}^{T_{\max }} D E M(T) \mathrm{d} T
$$

and

$$
T_{\text {mean }}=\frac{\int_{T_{\min }}^{T \max } D E M(T) T \mathrm{~d} T}{\int_{T \min }^{T \max } D E M(T) \mathrm{d} T} .
$$

The EM and temperature distribution maps of the flare region at 03:03 UT are plotted in Figure 3(b)-(c). It is seen that the hottest region in the cusp structure was located slightly lower than the cusp tip point, the presumed location of the MR, but higher than the above-the-loop-top HXR source. The center of the abovethe-loop-top HXR source was about $5^{\prime \prime}$ higher than the highest EM region. From the cusp tip to the hottest region, both the temperature and EM increased significantly. The hot cusp-like loops in the region, which were newly generated at the tip of the cusp through MR, had apparently a downward contracting motion, which is also know as flare loop shrinkage. Meanwhile, many downward small scale outflows appeared at the cusp tip in the $94 \AA$ and $131 \AA$ passbands (Figure 3(e)). Most of these outflows moved downward with initial velocities of $\sim 50-150 \mathrm{~km} \mathrm{~s}^{-1}$ and then stopped as they approached the top of the loop arcade. Notably, the outflows after 02:40 UT almost appeared to start at the similar height in the corona (Figure 3(e)). The dynamical evolution characteristics of the cool and hot plasma support the scenario of the MR concisely situated at the X-point.

Underneath the outflow region, there successively appeared a coronal 12-25 keV X-ray source and a $34 \mathrm{GHz}$ radio source, which are thought to be radiated from energetic electrons via bremsstrahlung and cyclotron emission mechanism, respectively. The energetic electrons streamed down along field lines, and bombarded the chromosphere, forming the brightenings seen in $1600 \AA$ and $1700 \AA$ passbands (Figure 3(a)). These observations further confirm the existence of MR above the cusp. It is worthy noting that this MR process is different from that during the main flare peak. Topologically, CLS and CLN were apparently impossible to participate in the MR underneath the MFR responsible for the main peak, since they were located above and in the periphery of the original MFR. Besides, the differences in the occurrence height and orientation between the post-flare arcade and the cusp also mean that the MR at the main peak and that at the second peak must take place at different locations. At the end of the flare, there appeared two groups of post-flare loop arcades at different heights (Figure 1(i)), which were the results of the two episodes of MR, respectively. 


\subsection{Three-dimensional topology of the magnetic reconnection at the magnetic null-point}

We have shown above the observational evidence of two-episode MR in a flare process. To further understand how MR takes place, we extrapolate the 3D coronal magnetic field based on the photospheric vector field observed by Helioseismic and Magnetic Imager (HMI; Scherrer et al.|2012; Schou et al. 2012) using a NLFFF model. The data are pre-processed into the Space-weather HMI Active Region Patches (SHARPs) heliographic Cylindrical Equal-Area (CEA) form (Bobra et al. 2014). Since the active region is rather quiet (no C-class flares and above) during the following two days after the flare, we use the vector magnetic field at 12:00:00 UT on 2012 November 10 (HARP 2186) as the bottom boundary for the extrapolation. The vector magnetic field of the active region shows a positive parasitic polarity (P1) encircled by three negative polarities located in the north, southeast, and west sides, respectively (N1, N2, and N3, in Figure 4(a)). Such a distribution is analogous to the lower surface of the magnetic 'dome' topology in Pontin et al. (2013). Through the NLFFF extrapolation, we also derive a similar dome-like structure, that is composed of the fan and spine structures associated with a null-point (Figure 4 (c)). Interestingly, the height of the null-point is $\sim 60 \mathrm{Mm}$ over the photosphere, almost the same as the height of the reconnection site of the second peak. Such a spatial coincidence indicates that the second-peak MR likely occurs around the magnetic null. We compare the magnetic field lines with the $171 \AA$ coronal loops around the cusp (Figure 4(c)-(d)) and find that they are mostly similar in topological configuration. Specifically, the CLS corresponds to one group of closed field lines connecting P1 and N2 (green lines in Figure 4(c)). The CLN has an identical location and orientation to the open field lines originating from N1 and passing nearby the null-point (yellow lines in Figure 4(c)). Both groups of field lines have sharp kink when they approach the magnetic null, outlining an X-shaped structure in projection, consistent with the $171 \AA$ X-shaped structure (Figure $1(\mathrm{~g})$ ). When they cross the magnetic null, their connectivities are supposed to change dramatically; in other words, magnetic field lines can 'reconnect' there. As a result, a new group of magnetic field lines - newly reconnected field lines are produced, which are, in EUV observations, the field lines shaping the hot cusp structure (red lines in Figure 4(c)). Since the reconnection takes place between the field lines in the spine and fan (Figure 4(c)), it probably corresponds to the spine-fan reconnection as Priest \& Titov (1996) and Pontin et al. (2013) defined.

\section{Discussion and conclusion}

In this work, we analyze the MR around a null-point in an M1.7 flare. The main results can be summarized as follows:

1. There existed two separate peaks in SXR and HXR time profiles resulted from MR in two different locations: one from the vertical CS created by the erupting MFR, and the other probably from the null-point roughly stationed above the pre-eruption MFR.

2. The magnetic field associated with the null-point went through two distinct steps: (1) separation distortion due to the throughput motion of the eruption/expanding MFR, and (2) inflow or recovery motion of the displaced field lines following the eruption.

Based on the above results, the two-step MR during the flare is schematically shown in Figure 5. During the flare impulsive phase, the MR (red star) takes place in the CS underneath the erupting MFR (red field lines) and produces the flare arcade 1(blue loops). As the MFR erupts through the MN, the magnetic field 
surrounding the null (green and yellow field lines) are pushed aside. When the MFR moves away, these distorted field lines begin to move back and produce the MR around the MN, produce the cusp (orange region) and flare arcade 2 (red loop).

In classical flare models that involve the current sheet, the reconnection site always keeps rising with time, due to the upward erupting MFR leading to the further stretching of the overlying magnetic field to a higher altitude. However, during the null-point reconnection, both the evolution of the above-the-loop-top hot region and the outflows indicated that the reconnection site (i.e. the magnetic null) almost keeps at the same altitude. However, the null-point reconnection also shows many similarities to the traditional flare reconnection, including the plasma inflow toward and outflow from the reconnection site, the flare cusp, the above-the-loop-top hot region, the HXR coronal source, and the post-flare loop arcade etc. Moreover, from the emission feature of the cusp, the most intensive emission region also lies somewhere outside the MR site (Krucker et al. 2010; Liu et al. 2013; Sun et al. 2014b).

Since magnetic nulls are rather common in the corona, they have the potential to trigger the reconnection there when encountered by an erupting MFR (Török et al. 2011), like what we observed in this event. This kind of MR may not be uncommon in solar flares, but is largely ignored in previous studies, mainly limited by observations.

Due to uncertainties in the magnetic field measurements, which are used as the bottom boundary, and the NLFFF modeling, we cannot completely rule out other MR regimes at flare second peak. However,

based on the above observations, we believe the null-point reconnection is a reasonable explanation. More features need to be explored theoretically and observationally to uncover the mechanism of the null-point reconnection in the future.

The authors thank the referee for constructive comments that helped improve the paper. This work was supported by NSFC under grants 11303016, 11373023, and NKBRSF under grant 2014CB744203. J. Zhang was supported by NSF AGS-1249270 and AGS-1460188.

\section{REFERENCES}

Baumann, G., Galsgaard, K., \& Nordlund, Å. 2013a, Sol. Phys., 284, 467

Baumann, G., Haugbølle, T., \& Nordlund, Å. 2013b, ApJ, 771, 93

Bobra, M. G., Sun, X., Hoeksema, J. T., Turmon, M., Liu, Y., Hayashi, K., Barnes, G., \& Leka, K. D. 2014, Sol. Phys., 289, 3549

Browning, P. K., Dalla, S., Peters, D., \& Smith, J. 2010, A\&A, 520, A105

Carmichael, H. 1964, NASA Special Publication, 50, 451

Chen, B., Bastian, T. S., Shen, C., Gary, D. E., Krucker, S., \& Glesener, L. 2015, Science, 350, 1238

Filippov, B. 1999, Sol. Phys., 185, 297

Fletcher, L., Metcalf, T. R., Alexander, D., Brown, D. S., \& Ryder, L. A. 2001, ApJ, 554, 451

Galsgaard, K., \& Pontin, D. I. 2011, A\&A, 529, A20 
Hannah, I. G., \& Kontar, E. P. 2012, A\&A, 539, A146

Hirayama, T. 1974, Sol. Phys., 34, 323

Kopp, R. A., \& Pneuman, G. W. 1976, Sol. Phys., 50, 85

Krucker, S., Hudson, H. S., Glesener, L., White, S. M., Masuda, S., Wuelser, J.-P., \& Lin, R. P. 2010, ApJ, 714,1108

Lemen, J. R., et al. 2012, Sol. Phys., 275, 17

Lin, J., \& Forbes, T. G. 2000, J. Geophys. Res., 105, 2375

Lin, R. P., et al. 2002, Sol. Phys., 210, 3

Liu, W., Chen, Q., \& Petrosian, V. 2013, ApJ, 767, 168

Masuda, S., Kosugi, T., Hara, H., Tsuneta, S., \& Ogawara, Y. 1994, Nature, 371, 495

McKenzie, D. E., \& Hudson, H. S. 1999, ApJ, 519, L93

Nakajima, H., et al. 1994, IEEE Proceedings, 82, 705

Pesnell, W. D., Thompson, B. J., \& Chamberlin, P. C. 2012, Sol. Phys., 275, 3

Pontin, D. I., \& Galsgaard, K. 2007, Journal of Geophysical Research (Space Physics), 112, A03103

Pontin, D. I., Priest, E. R., \& Galsgaard, K. 2013, ApJ, 774, 154

Priest, E. R., \& Pontin, D. I. 2009, Physics of Plasmas, 16, 122101

Priest, E. R., \& Titov, V. S. 1996, Philosophical Transactions of the Royal Society of London Series A, 354, 2951

Scherrer, P. H., et al. 2012, Sol. Phys., 275, 207

Schou, J., et al. 2012, Sol. Phys., 275, 229

Stanier, A., Browning, P., \& Dalla, S. 2012, A\&A, 542, A47

Sturrock, P. A. 1966, Nature, 211, 695

Su, Y., Veronig, A. M., Holman, G. D., Dennis, B. R., Wang, T., Temmer, M., \& Gan, W. 2013, Nature Physics, 9, 489

Sui, L., \& Holman, G. D. 2003, ApJ, 596, L251

Sun, J. Q., Cheng, X., Guo, Y., Ding, M. D., \& Li, Y. 2014a, ApJ, 787, L27

-. 2014b, ApJ, 787, L27

Sun, J. Q., et al. 2015, Nature Communications, 6, 7598

Sun, X., Hoeksema, J. T., Liu, Y., Chen, Q., \& Hayashi, K. 2012, ApJ, 757, 149

Svestka, Z., \& Cliver, E. W. 1992, in Lecture Notes in Physics, Berlin Springer Verlag, Vol. 399, IAU Colloq. 133: Eruptive Solar Flares, ed. Z. Svestka, B. V. Jackson, \& M. E. Machado, 1 
Török, T., Panasenco, O., Titov, V. S., Mikić, Z., Reeves, K. K., Velli, M., Linker, J. A., \& De Toma, G. 2011, ApJ, 739, L63

Wang, H., \& Liu, C. 2012, ApJ, 760, 101

Wyper, P. F., Jain, R., \& Pontin, D. I. 2012, A\&A, 545, A78

Xiao, C. J., et al. 2006, Nature Physics, 2, 478

Yokoyama, T., Akita, K., Morimoto, T., Inoue, K., \& Newmark, J. 2001, ApJ, 546, L69

Zhang, J., Dere, K. P., Howard, R. A., Kundu, M. R., \& White, S. M. 2001, ApJ, 559, 452

Zhu, C., Liu, R., Alexander, D., \& McAteer, R. T. J. 2016, ApJ, 821, L29 


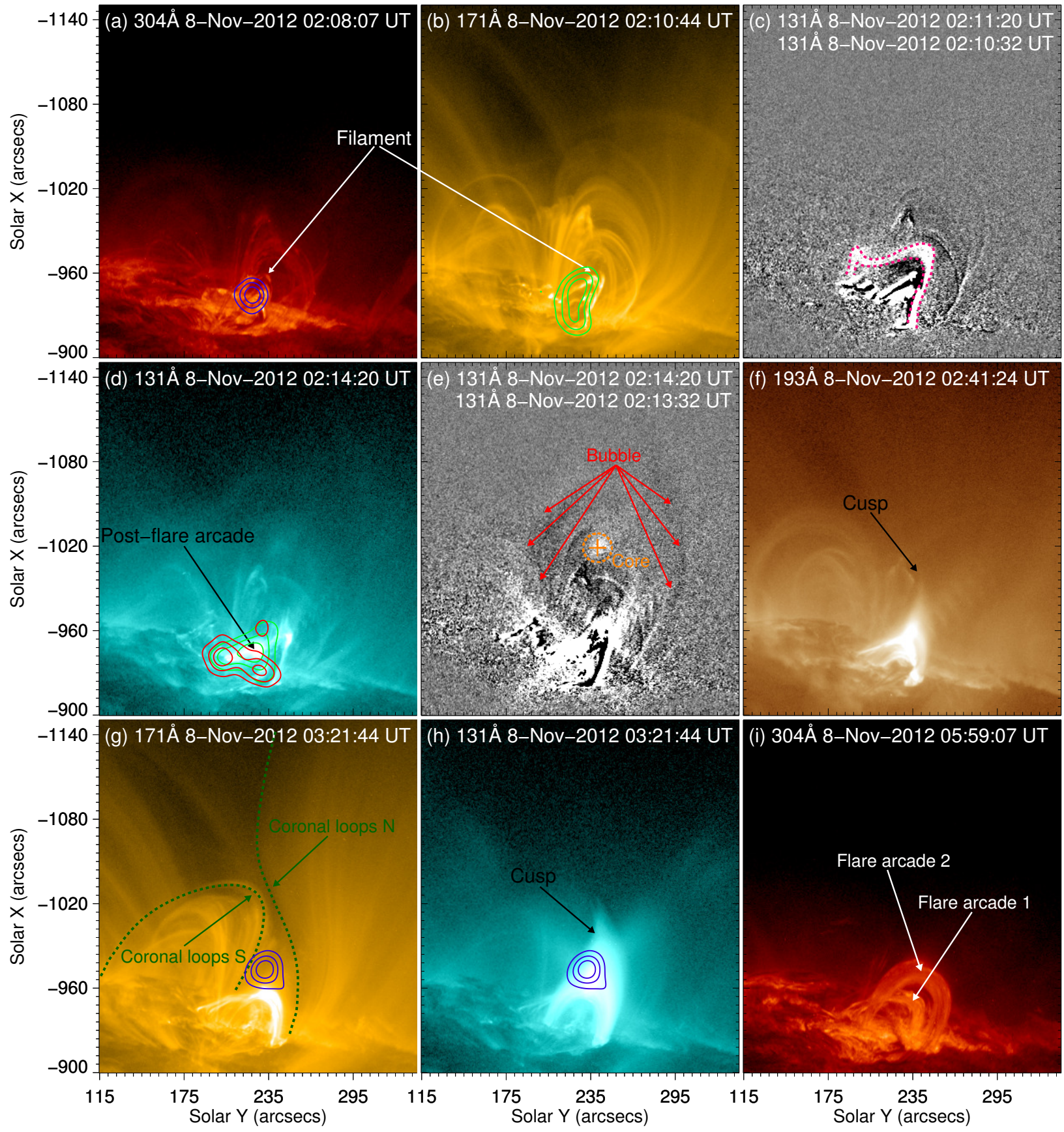

Fig. 1.- Overview of the flare evolution. (a)-(b) AIA $304 \AA$ and $171 \AA$ images of the flare region at 02:08:07 UT and 02:10:44 UT, respectively. (c) AIA $131 \AA$ difference map between 02:11:20 UT and 02:10:32 UT. The pink dashed lines sketch a possible rising MFR. (d) AIA 131 A image of the flare region at 02:14:20 UT. (e) AIA $131 \AA$ difference map between 02:14:20 UT and 02:13:32 UT, showing an intense core surrounded by a bubble. (f) AIA $193 \AA$ image of the flare region at 02:41:24 UT, revealing a high-lying cusp. (g) AIA $171 \AA$ image of the flare region at 03:21:44 UT, the two cyan dashed lines sketch two groups of coronal loops that probably participating in the second-peak reconnection. (h) AIA $131 \AA$ image at 03:21:44 UT showing the cusp. (i) AIA $304 \AA$ image at 05:59:07 UT. The over-plotted contours are RHESSI 6-12 $\mathrm{keV}$ (blue), 12-25 keV (green), and 25-50 keV (red) X-ray sources at levels of 40\%, 60\%, and $80 \%$. (Animations are available in the online journal.) 


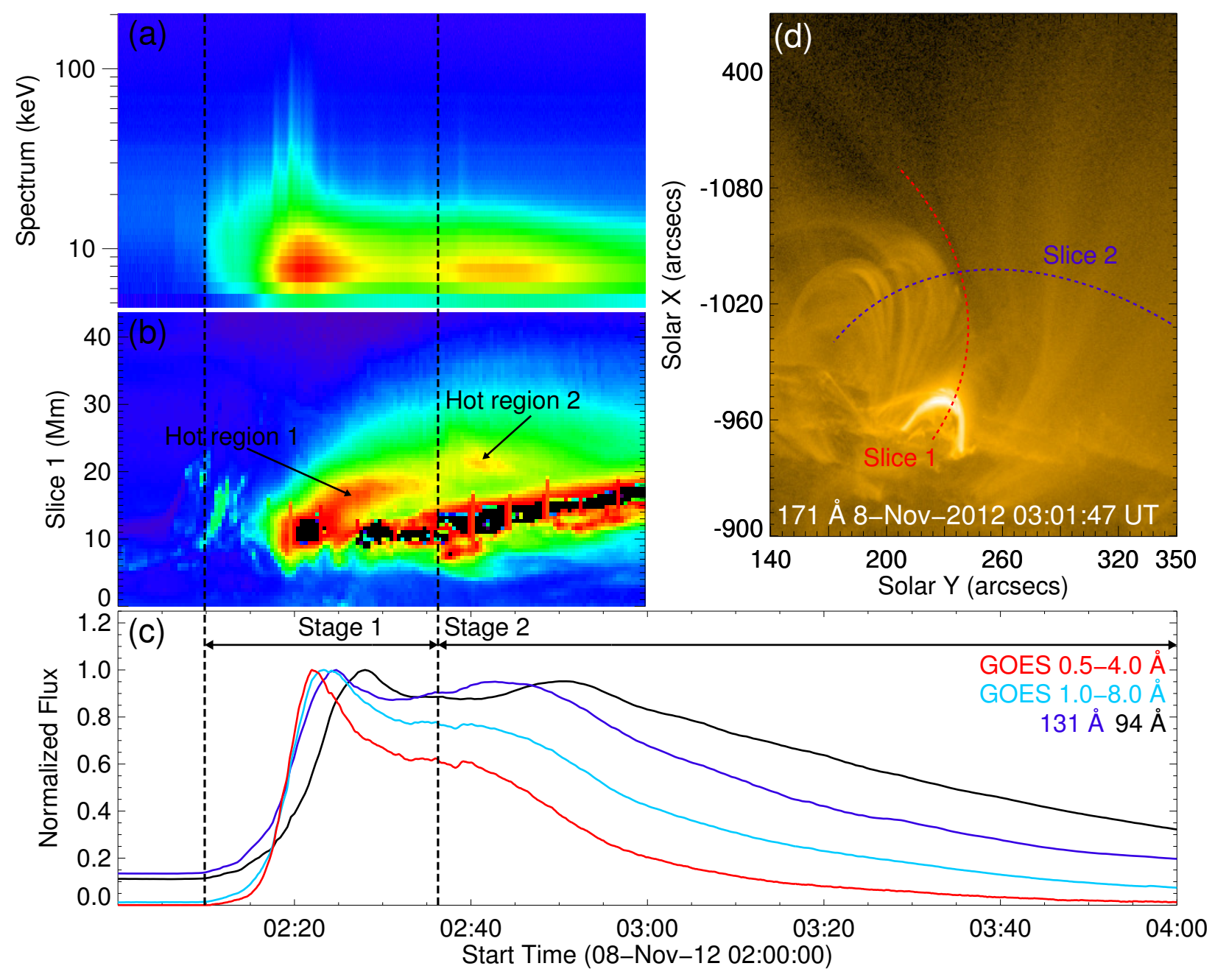

Fig. 2.- Two episodes of energy release in the flare. (a) Fermi $4.2-200 \mathrm{keV}$ spectrogram. (b) Timeslice evolution of $193 \AA$ emission along the flare cusp. (c) Time profiles of the GOES SXR, AIA $94 \AA$, and $131 \AA$ flux of the flare region normalized to the maximum values. (d) AIA $171 \AA$ image of the flare region at 03:01:47 UT. The blue and red dashed lines denote the slice 1 and 2 used for Figures 3(b), 4(d)-(e). The black dashed lines separate the flare evolution into two stages. 

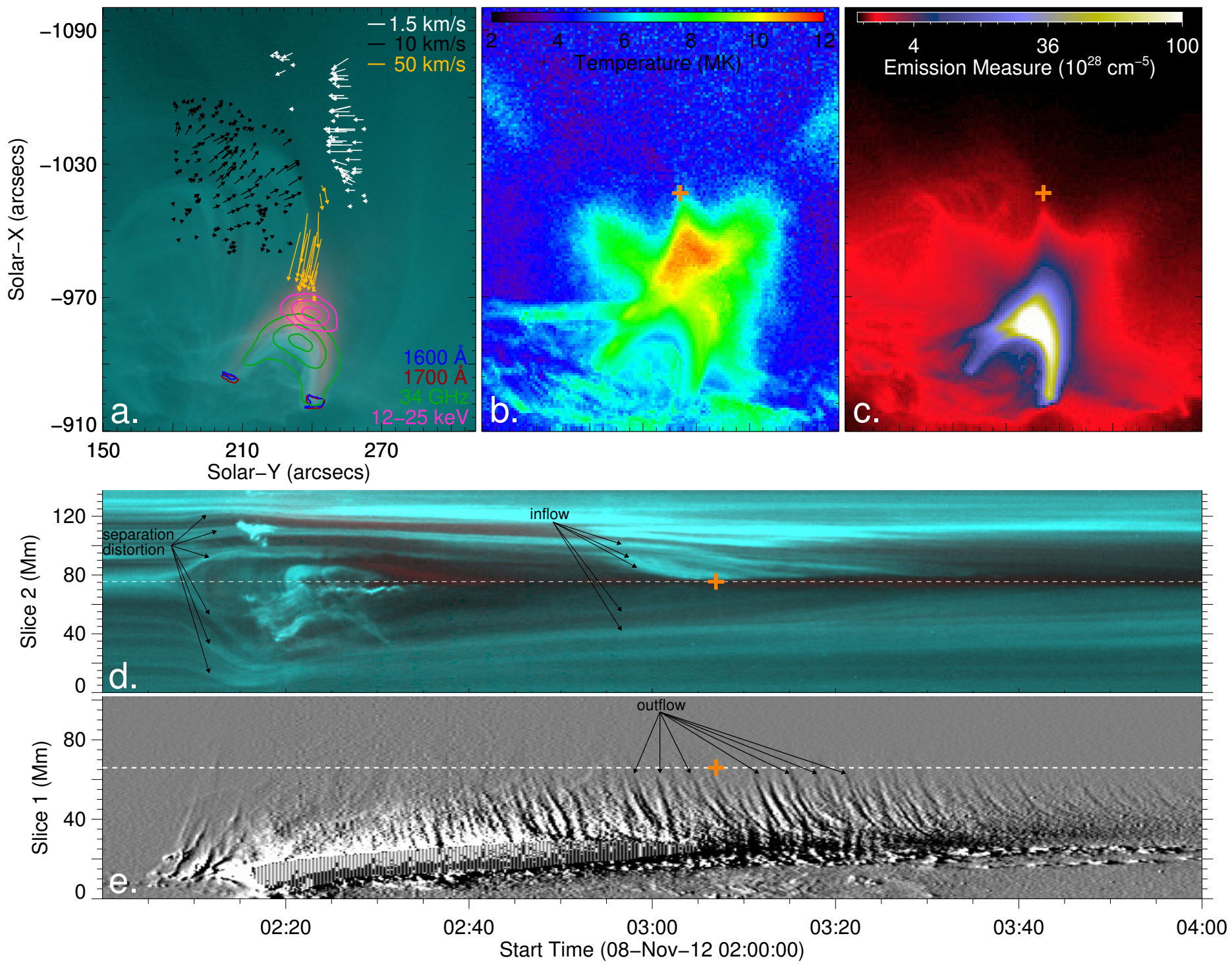

Fig. 3.- Observational evidence of MR around a 3D null-point. (a) A composite image of the AIA $131 \AA$ (red) and $171 \AA$ (cyan) passbands at 03:03 UT. The arrows scaled by the white, black, and orange line segments are inflow and outflow plasma velocities around 03:03 UT and 03:17 UT, respectively. The over-plotted contours in pink, green, blue, and red indicate the emission of $12-25 \mathrm{keV}(50 \%, 70 \%$, and $90 \%), 34 \mathrm{GHz}(30 \%, 60 \%$, and 90\%), $1600 \AA$ (50\%), and $1700 \AA$ (50\%) around 03:03 UT, respectively. (b) DEM-weighted temperature map (c) EM map of the flare region. (d) $131 \AA$ (red) and $171 \AA$ (cyan) time evolution along Slice 2. (e) $131 \AA$ A time-difference evolution along Slice 1. The orange crosses in panel (b)-(e) denote the same position, which is the suspected reconnection site. 


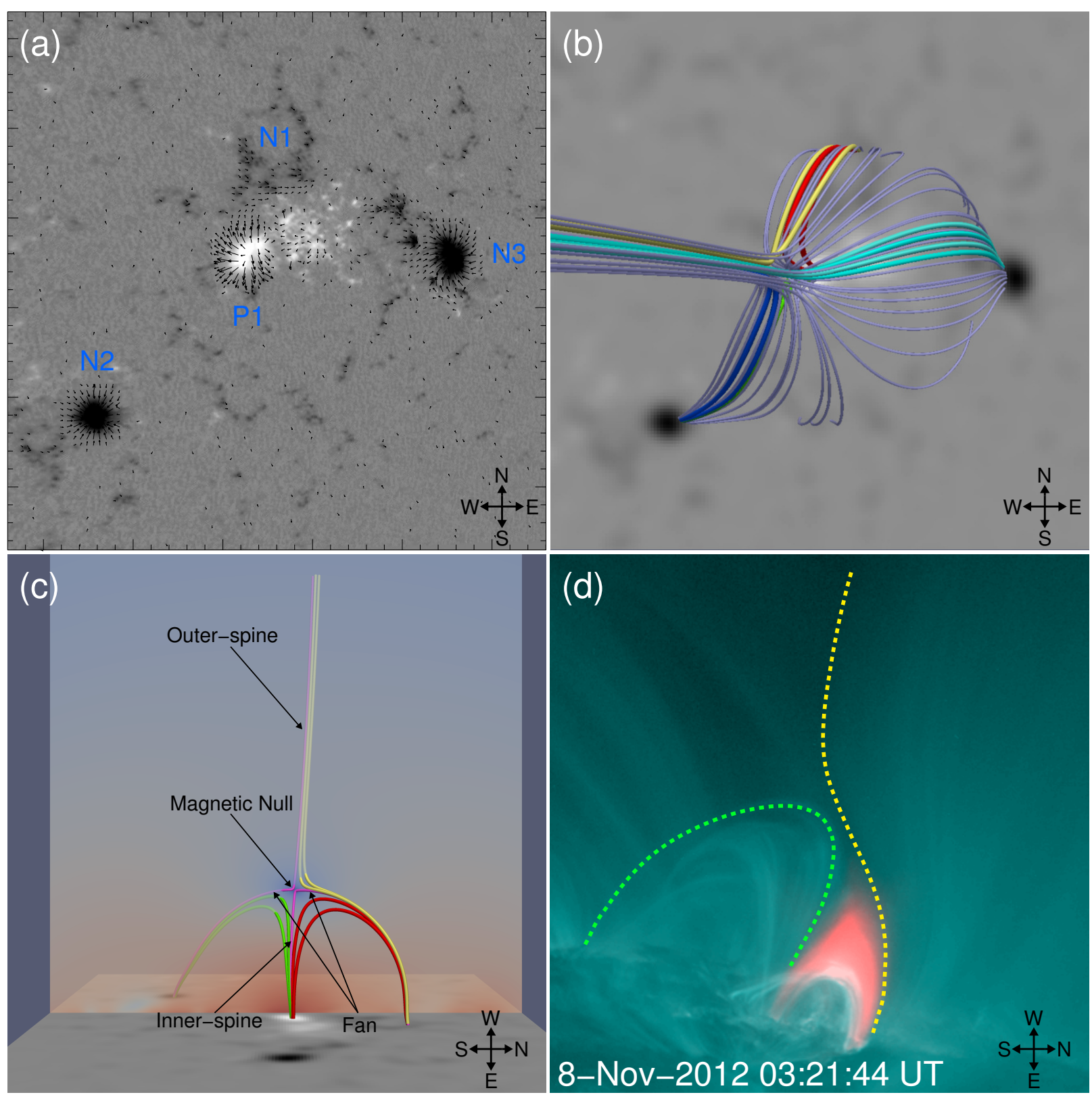

Fig. 4.- The magnetic field topology of the active region. (a) Photosphere magnetic field of the active region. 'N1', 'N2', 'N3', and 'P1' denote three negative polarities and one positive polarity, respectively. (b) A top view of the magnetic field lines in the active region. (c) Magnetic fields participating in the second peak null-point reconnection. The vertical cut showing the magnetic intensity map in which a minimum region corresponds to a magnetic null. The spine and fan lines are denoted and plotted in pink. (d) The AIA $131 \AA$ and $171 \AA$ composite image. The green and yellow dashed lines sketch the coronal loops participate in the null-point reconnection, which are also used to show a similarity to the green and yellow field lines in panel (c).

(Animation is available in the online journal.) 

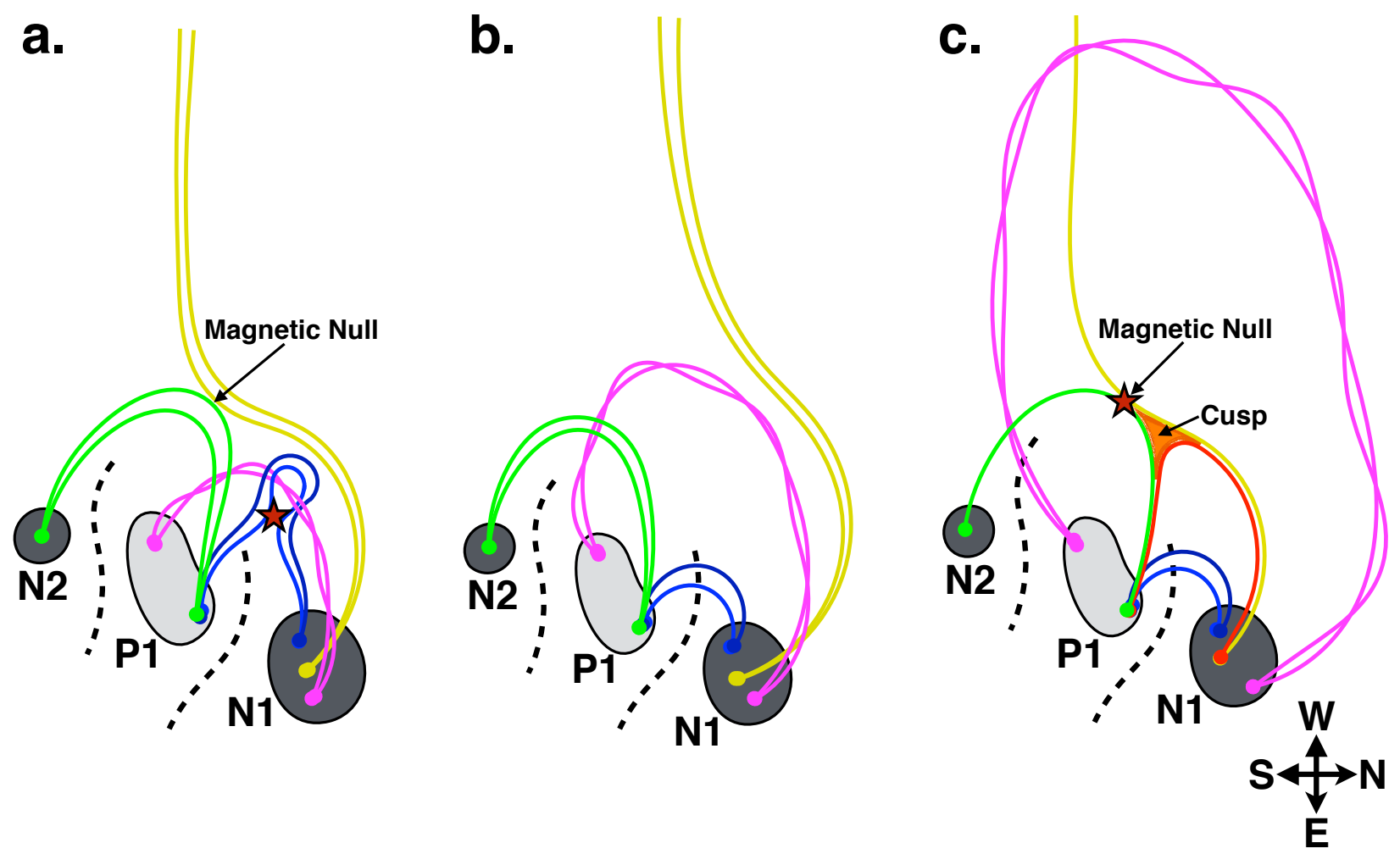

Fig. 5.- Schematic drawing of the two-step MR involved in the dual-peak flares. MR in the CS under the MFR is triggered when the MFR erupts upward (a). Meanwhile, the MFR distorts and separates the magnetic fields around the null-point (b), which, however begin to recover toward the null when the MFR leaves away and further causes the reconnection around the null (c). 